\title{
Variations of posterior vitreous detachment
}

\author{
Akihiro Kakehashi, Masanori Kado, Jun Akiba, Hiroyuki Hirokawa
}

\begin{abstract}
Aims-To identify variations in posterior vitreous detachment (PVD) and establish a clinical classification system for PVD.

Methods-400 consecutive eyes were examined using biomicroscopy and vitreous photography and classified the PVD variations-complete PVD with collapse, complete PVD without collapse, partial PVD with thickened posterior vitreous cortex (TPVC), or partial PVD without TPVC.
\end{abstract}

Results-In each PVD type, the most frequently seen ocular pathologies were as follows: in complete PVD with collapse (186 eyes), age related changes without vitreoretinal diseases ( 77 eyes, $41.4 \%$ ) and high myopia (55 eyes, $29.6 \%$ ); in complete PVD without collapse (39 eyes), uveitis (23 eyes, $59.0 \%$ ) and central retinal vein occlusion (8 eyes, 20.5\%); in partial PVD with TPVC (64 eyes), proliferative diabetic retinopathy ( 30 eyes, $46.9 \%$ ); and in partial PVD without TPVC (111 eyes), age related changes without vitreoretinal diseases (62 eyes, 55.9\%). This PVD categorisation was significantly associated with the prevalence of each vitreoretinal disease $\left(p<0.0001, \chi^{2}\right.$ test on contingency table).

Department of Ophthalmology, Omiya Medical Center, Jichi Medical School, Omiya, Japan Akihiro Kakehashi

Department of Ophthalmology, Asahikawa Medical College, Asahikawa, Japan

M Kado

J Akiba

H Hirokawa

Correspondence to: Akihiro Kakehashi, MD, Department of Ophthalmology, Omiya Medical Center, Jichi Medical School, 1-847 Amanuma-cho, Omiya, 330 Japan.

Accepted for publication 12 February 1997
Conclusions-PVD variations can be classified into four types, which is clinically useful because each type corresponds well to specific vitreoretinal changes.

(Br f Ophthalmol 1997;81:527-532)

Results of previous studies have shown that characteristic types of posterior vitreous detachment (PVD) are observed with specific vitreoretinal diseases. For example, partial PVD is a characteristic vitreous change in proliferative diabetic retinopathy, ${ }^{1-4}$ and complete PVD is observed in typical age related PVD. ${ }^{5-12}$ The morphological configurations of PVD vary, and the variations strongly affect the prognosis of the vitreoretinal diseases. For example, preretinal neovascularisation in diabetic retinopathy rarely occurs when there is complete PVD. ${ }^{12}{ }^{13}$ However, the causes of these PVD variations are unknown. Therefore, we classified the PVD types found in the clinical cases and correlated the various vitreoretinal diseases associated with these PVD types.

\section{Patients and methods}

To clarify the typical vitreous changes occurring in retinal diseases, we examined 400 consecutive eyes with PVD (321 patients; mean age 58.6 years, SD 16.6) by biomicroscopy and vitreous photography using the El BayadiKajiura preset lens, the +90 dioptre preset lens, or both. ${ }^{14}{ }^{15}$ This technique provides strong illumination, a large illumination observation angle and image, and minimal distortion and glare. Although dynamic movement of the vitreous can be observed through a contact lens, the technique using these positive preset lenses permits dynamic observation of the vitreous more easily and is more comfortable for the patients. These patients had been referred to the department of vitreous study at Asahikawa Medical College for further evaluation of vitreoretinal changes.

The following ophthalmic conditions were seen in the 400 study eyes: age related change without significant vitreoretinal disease, 141 eyes (104 patients; mean age 67.5 (SD 8.0) years); uveitis, 74 eyes ( 55 patients; mean age 47.3 (20.1) years); high myopia (refractive error greater than -8.0 dioptres), 66 eyes (60 patients; mean age 51.2 (17.1) years); proliferative diabetic retinopathy, 33 eyes (29 patients, mean age 52.8 (17.6) years); nonproliferative diabetic retinopathy, 23 eyes (17 patients; mean age 66.4 (11.6) years); branch retinal vein occlusion, 27 eyes (25 patients; mean age 66.3 (11.6) years); central retinal vein occlusion, 20 eyes (20 patients, mean age 59.5 (16.7) years); retinal breaks, nine eyes (seven patients, mean age 52.7 (16.1) years); and retinitis pigmentosa, seven eyes (four patients, mean age 48.8 (19.3) years).

The morphological variations of PVD were classified into four types:

(1) Complete PVD with collapse, in which the vitreous gel exhibits liquefaction and, therefore, a large retrocortical space and smooth movement of the detached vitreous with ocular movement are observed. In this type, the posterior vitreous cortex has a characteristically sigmoidal shape when the patient is sitting (Fig 1). Weiss's ring is typically observed on the posterior vitreous cortex. 
A
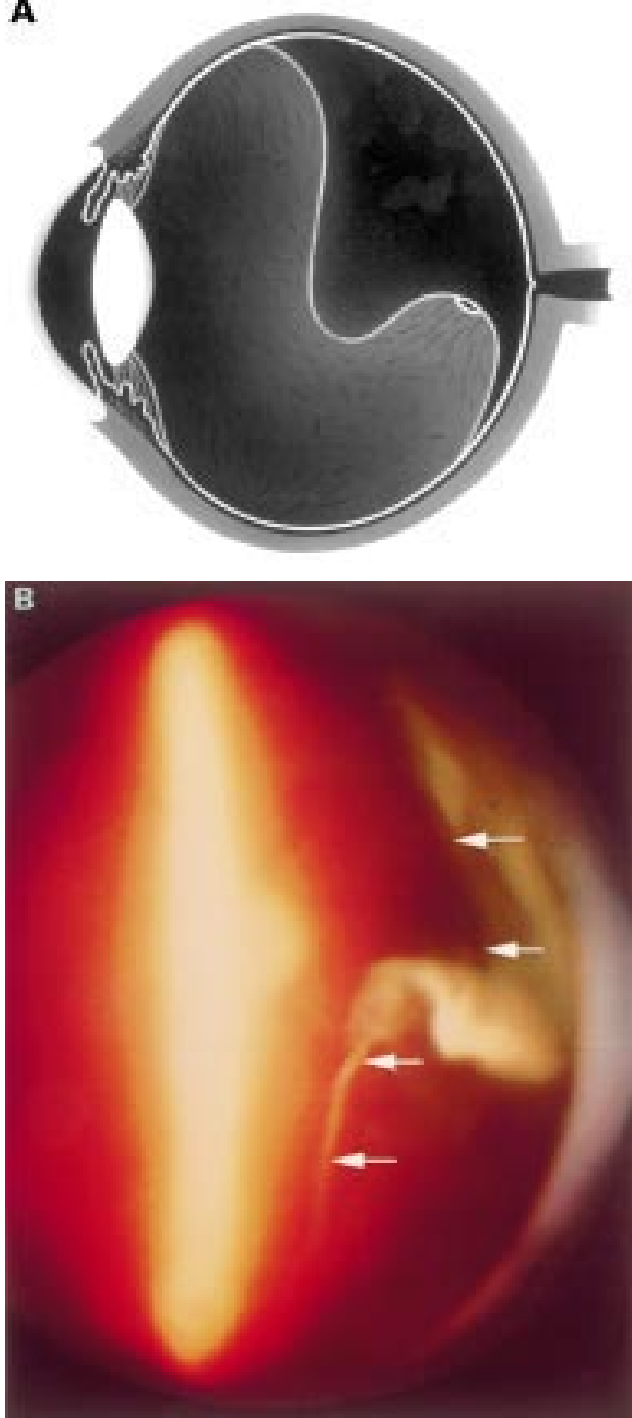

Figure 1 (A) Schematic sketch of complete posterior vitreous detachment (PVD) with collapse. The posterio vitreous cortex has a characteristically sigmoidal shape. (B) In a patient who complained of floaters in his visual field, the biomicroscopic vitreous examination revealed complete PVD. The vitreous gel was mildly condensed and, with ocular movement, the posterior vitreous cortex (arrows) oscillated in a smooth, wavy motion. This case was classified as complete PVD with collapse.

(2) Complete PVD without collapse, in which the vitreous gel is minimally liquefied and, therefore, movement of the detached vitreous is limited. When vitreous shrinkage is advanced, the retrocortical space is clearly defined, and the posterior vitreous cortex is convex (Fig 2). Weiss's ring is also typically observed on the posterior vitreous cortex.

(3) Partial PVD with a thickened posterior vitreous cortex (TPVC), in which the detached posterior vitreous cortex is thickened, taut, and anchored at two points (Fig 3). Some variations in this category are seen, because the two points of attachment vary - that is, at the vitreous base, the optic disc, along the vascular arcade, the neovascular complex, and the macula.

(4) Partial PVD without TPVC, in which the posterior vitreous cortex is not thickened
A
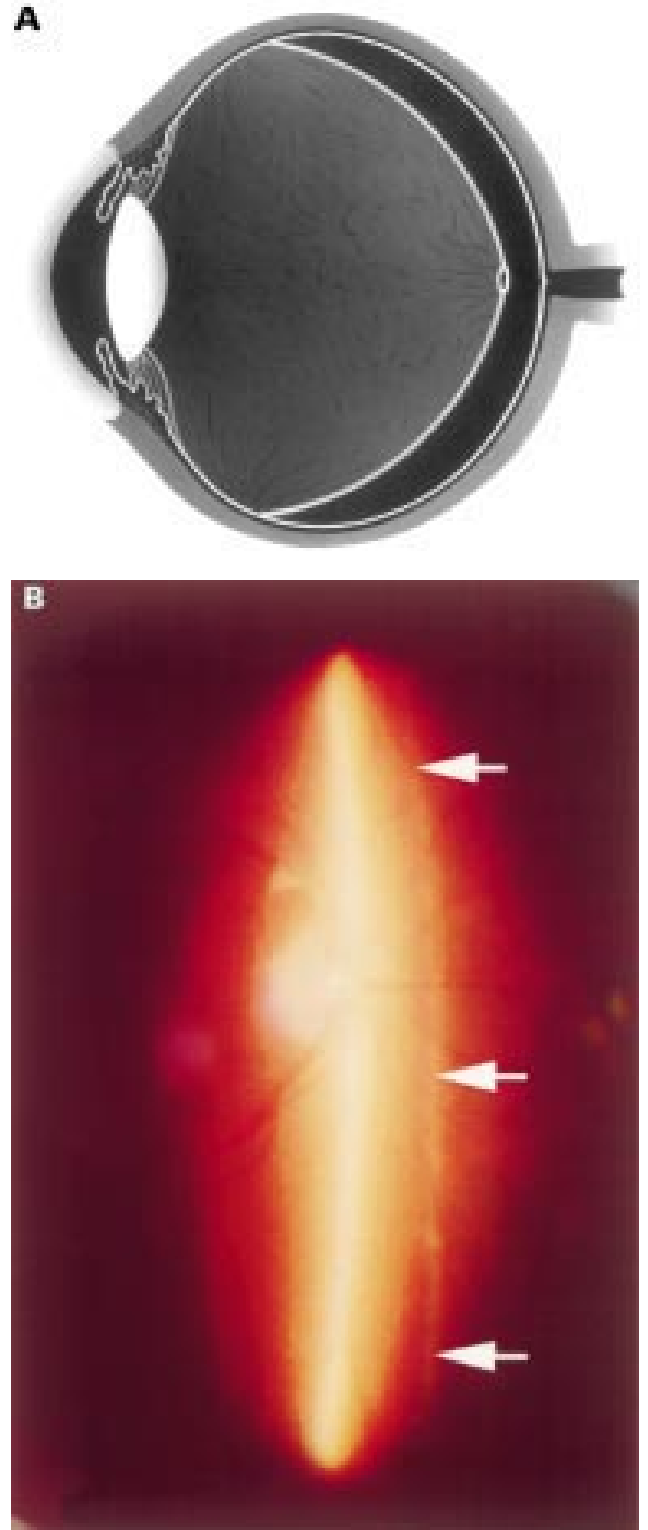

Figure 2 (A) Schematic sketch of complete posterior vitreous detachment (PVD) without collapse. The posterior vitreous cortex is convex and shallowly detached. (B) In a patient with uveitis, the posterior vitreous cortex (arrows) is shallowly detached from the retina; vitreous opacities are observed in the vitreous gel and the posterior vitreous cortex is convex. The vitreous gel is condensed and has minimal mobility even with rapid ocular movement. This case was classified as complete PVD without collapse.

(Fig 4). In the last category, we also included vitreous gel attachment to the macular area through a round defect in the posterior vitreous cortex (Fig 5). This pathology was first described by Jaffe, ${ }^{9}$ characterised and photographed by Sebag and Balazs, ${ }^{16}{ }^{17}$ and documented photographically and videographically in clinical cases by Kakehashi and associates. ${ }^{18} 19$

The $\chi^{2}$ test and the Student's $t$ test were used for data analysis. The level of statistical significance was $\mathrm{p}<0.05$.

\section{Results}

The mean patient ages in years in each group were as follows: complete PVD with collapse, 61.6; complete PVD without collapse, 44.6; 

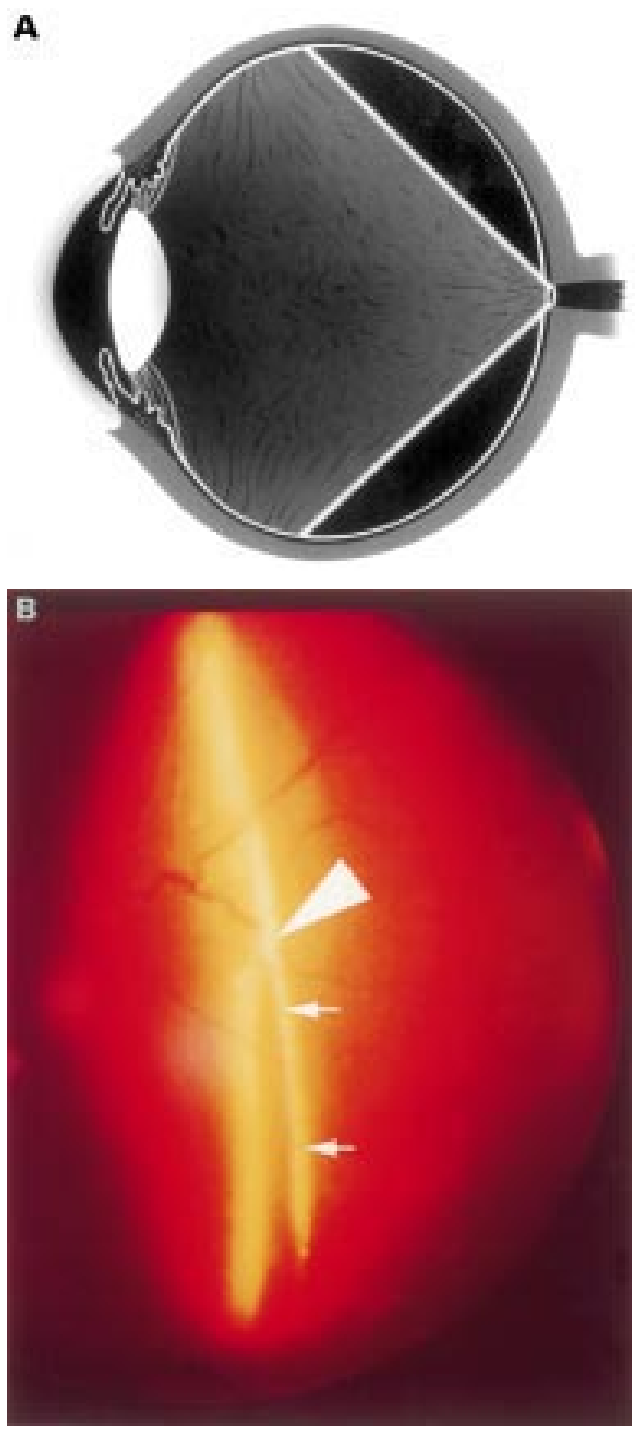

Figure 3 (A) Schematic sketch of partial posterior vitreous detachment (PVD) with a thickened posterior vitreous cortex (TPVC), which exhibits minimal movement with ocular movement. (B) In a patient with proliferative diabetic retinopathy, the fundus examination by indirect ophthalmoscopy revealed tractional retinal detachment. Biomicroscopic vitreous examination revealed vitreous traction upon the detached retina (arrowhead) with a condensed posterior vitreous cortex (arrows). This case was classified as partial PVD with TPVC.

partial PVD with TPVC, 51.9; and partial PVD without TPVC, 62.5. The mean patient ages were significantly higher in patients with complete PVD with collapse and partial PVD without TPVC than in the other groups ( $\mathrm{p}=0.0001$ by the Student's $t$ test).

Table 1 shows the prevalence of ocular disease in each PVD type. All cases in this study were included in this table. The PVD categorisation was statistically significantly associated with the prevalence of each vitreoretinal disease $(\mathrm{p}<0.0001$, by goodness test of fit for $\chi^{2}, 4 \times 9$ contingency table). In each PVD type, the most frequently seen ocular pathologies were as follows: in complete PVD with collapse (186 eyes), age related change without vitreoretinal diseases (77 eyes, $41.4 \%$ ) and high myopia (55 eyes, $29.6 \%$ ); in complete PVD without collapse (39 eyes), uveitis (23
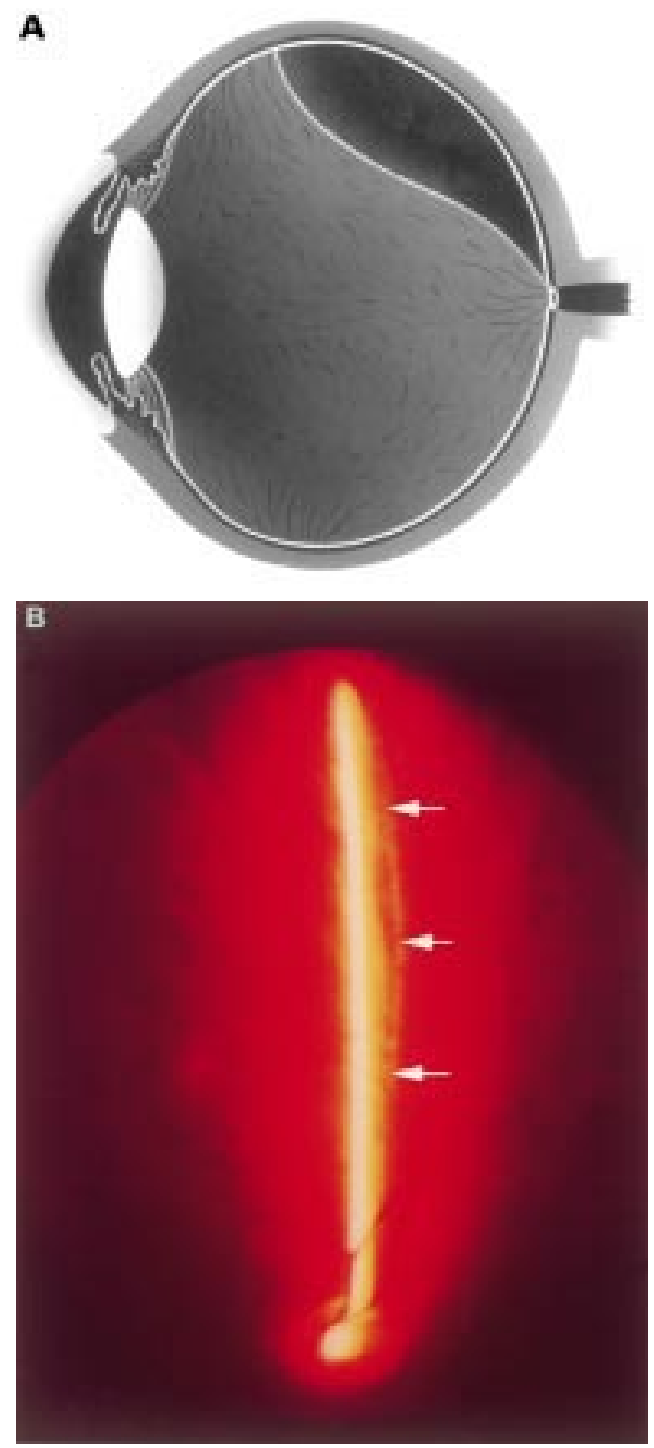

Figure 4 (A) Schematic sketch of partial posterior vitreous detachment (PVD) without a thickened posterior vitreous cortex (TPVC), which exhibits some mobility with ocular movement. (B) In a patient who complained of flashes in his right eye, the fundus examination by indirect ophthalmoscopy did not reveal retinal disease. The biomicroscopic vitreous examination showed localised PVD in the upper quadrants of the right eye. The vitreous gel was moderately liquefied, and with ocular movement, the posterior vitreous cortex (arrows) was not condensed and had a smooth, wavy motion. This case was classified as partial PVD without TPVC.

eyes, $59.0 \%$ ) and central retinal vein occlusion (eight eyes, 20.5\%); in partial PVD with TPVC (64 eyes), proliferative diabetic retinopathy (30 eyes, $46.9 \%)$; and in partial PVD without TPVC (111 eyes), age related change without vitreoretinal diseases (62 eyes, 55.9\%). The prevalence of ocular disease was statistically different among the PVD types $(p<0.0001$, between complete PVD with collapse and complete PVD without collapse, complete PVD with collapse and partial PVD with TPVC, complete PVD without collapse and partial PVD with TPVC, partial PVD with TPVC and partial PVD without TPVC; $\mathrm{p}<0.001$, complete PVD with collapse and partial PVD without TPVC). 

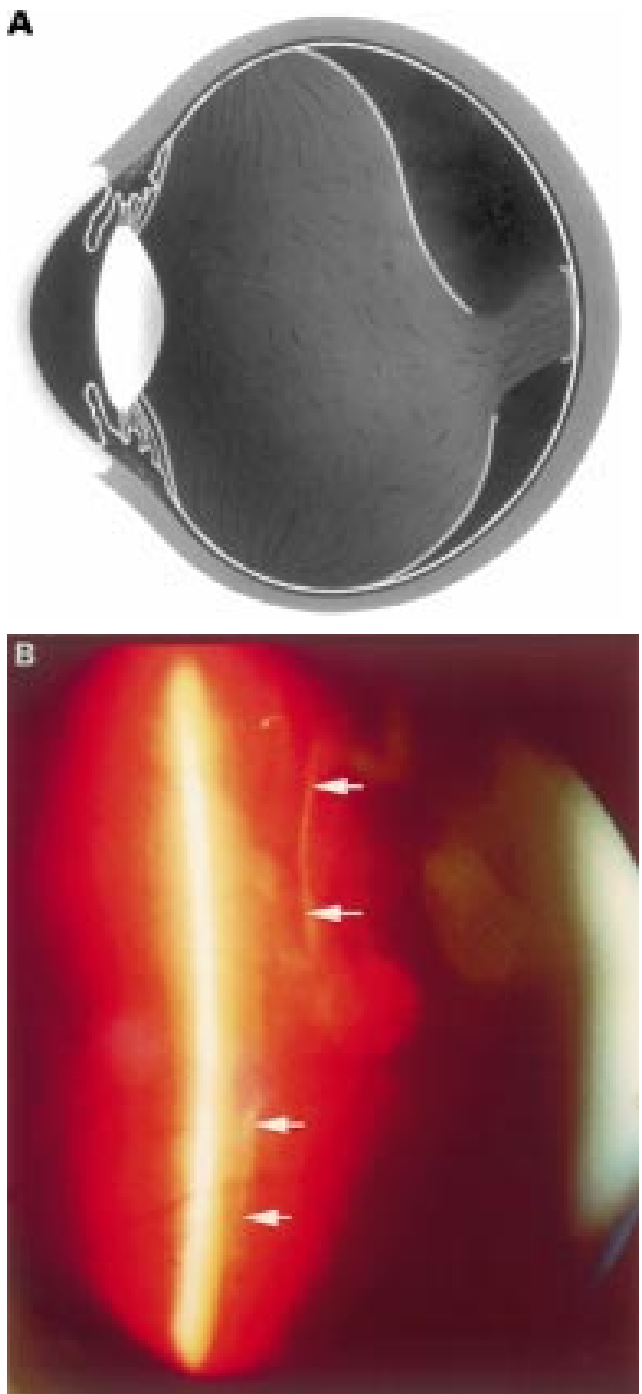

Figure 5 (A) Schematic sketch of another type of partial posterior vitreous detachment (PVD) without a thickened posterior vitreous cortex (TPVC). The vitreous gel was attached to the macular area through a round defect in the posterior vitreous cortex. (B) In a patient who complained of floaters in his visual field, the fundus examination by indirect ophthalmoscopy did not reveal retinal disease. The biomicroscopic vitreous examination revealed complete PVD except at the macular area. The vitreous gel remained attached to the macular area through a round defect in the posterior vitreous cortex. Upon ocular movement, the posterior vitreous cortex (arrows) was not condensed and had a smooth, wavy motion. This case also was classified as partial PVD without TPVC.
The prevalence of each PVD type in common vitreoretinal diseases found in the present study is shown in Table 2. The prevalence of each PVD type in each common vitreoretinal disease was statistically significantly different except for that found between uveitis and central retinal vein occlusion $(p>0.5)$, between branch retinal vein occlusion and non-proliferative diabetic retinopathy $(p>0.1)$, and between non-proliferative diabetic retinopathy and central retinal vein occlusion $(p>0.1)$. In other words, uveitis and central retinal vein occlusion had a similar pattern of the prevalence of each PVD.

\section{Discussion}

Some PVD classifications have been proposed previously, the most practical of which was suggested by Tolentino and colleagues. ${ }^{8}$ These authors classified PVD based on whether or not detachment of the posterior vitreous cortex is complete or incomplete and if the detached cortex collapsed. ${ }^{8}$ They also classified atypical PVD into three types, funnel-shaped, hammock-shaped, and PVD with a dehiscent cortex.

Even though their classification accurately represents the PVD variations, it does not correspond well to specific vitreoretinal changes. For example, both partial PVD with a condensed posterior vitreous cortex resulting from proliferative diabetic retinopathy and partial PVD without a condensed posterior vitreous cortex resulting from age related changes are classified as incomplete PVD without collapse. Therefore, we refined the old classification and made a more precise classification and correlated it with specific vitreoretinal changes.

Three main factors are associated with PVD - that is, vitreous liquefaction, vitreous shrinkage, and weakening of vitreoretinal adhesion. Vitreous liquefaction frequently is observed in association with age related vitreous changes and in highly myopic eyes. The mechanism of age or disease related vitreous liquefaction is uncertain. However, some investigators have suggested that free radicals cause hyaluronan depolymerisation which leads to destruction of the gel structure (liquefaction).$^{20-22}$ Other studies showed that in

Table 1 Prevalence of ocular diseases by posterior vitreous detachment (PVD) (no of eyes (\%))

\begin{tabular}{lcccrr}
\hline & CPVD with collapse & CPVD without collapse & PPVD with TPVC & PPVD without TPVC & Total \\
\hline Age $^{\star}$ & $77(41.4)$ & $1(2.6)$ & $1(1.6)$ & $62(55.9)$ & 141 \\
Uveitis & $21(11.3)$ & $23(59.0)$ & $18(28.1)$ & $12(10.8)$ & 74 \\
High myopia & $55(29.6)$ & $2(5.1)$ & $1(1.6)$ & $3(2.2)$ & 66 \\
PDR & $0(0)$ & $0(0)$ & $10(46.9)$ & $8(7.2)$ & 33 \\
BRVO & $8(4.3)$ & $1(2.6)$ & $2(3.6)$ & $8(7.2)$ & 27 \\
NPDR & $11(5.9)$ & $8(5.1)$ & $2(3.1)$ & $4(3.6)$ & 23 \\
CRVO & $6(3.2)$ & $2(5.1)$ & $0(0)$ & $4(3.8)$ & 20 \\
Retinal break & $5(2.7)$ & $0(0)$ & 64 & 111 & 9 \\
RP & $3(1.6)$ & 39 & & & 4 \\
Total & 186 & & & & 400
\end{tabular}

*Age indicates age related change without evidence of vitreoretinal disease.

$\mathrm{CPVD}=$ complete posterior vitreous detachment; PPVD = partial posterior vitreous detachment; TPVC $=$ thickened posterior vitreous cortex; PDR = proliferative diabetic retinopathy; $\mathrm{NPDR}=$ non-proliferative diabetic retinopathy; $\mathrm{BRVO}=$ branch retinal vein occlusion; $\mathrm{CRVO}=$ central retinal vein occlusion; $\mathrm{RP}=$ retinitis pigmentosa .

p $<0.0001$; CPVD with collapse - CPVD without collapse; CPVD with collapse - PPVD with TPVC; CPVD without collapse - PPVD with TPVC; CPVD without collapse - PPVD without TPVC; PPVD with TPVC - PPVD without TPVC. $\mathrm{p}<0.001$; CPVD with collapse - PPVD without TPVC. 
Table 2 Prevalence of posterior vitreous detachment (PVD) by ocular diseases (no of eyes (\%))

\begin{tabular}{lcccrr}
\hline & CPVD with collapse & CPVD without collapse & PPVD with TPVC & PPVD without TPVC & Total \\
\hline Age $^{\star}$ & $77(54.6)$ & $1(0.7)$ & $1(0.7)$ & $62(44.0)$ & 141 \\
Uveitis & $21(28.4)$ & $23(31.3)$ & $18(24.3)$ & $12(16.2)$ & 74 \\
High myopia & $55(83.3)$ & $2(3.0)$ & $1(1.5)$ & $8(12.2)$ & 66 \\
PDR & $0(0)$ & $0(0)$ & $30(90.1)$ & $3(9.9)$ & $8(29.6)$ \\
BRVO & $8(29.6)$ & $1(3.7)$ & $2(8.7)$ & $8(34.8)$ & 27 \\
NPDR & $11(47.8)$ & $8(8.7)$ & $2(10.0)$ & $4(20.0)$ & 23 \\
CRVO & $6(30.0)$ & $8(40.0)$ & & & 20 \\
\hline
\end{tabular}

*Age indicates age related change without evidence of vitreoretinal disease.

$\mathrm{CPVD}=$ complete posterior vitreous detachment; PPVD = partial posterior vitreous detachment; $\mathrm{TPVC}=$ thickened posterior vitreous cortex; $\mathrm{PDR}$ = proliferative diabetic retinopathy; $\mathrm{NPDR}=$ non-proliferative diabetic retinopathy; $\mathrm{BRVO}=$ branch retinal vein occlusion; $\mathrm{CRVO}=$ central retinal vein occlusion

p<0.0001; aged - uveitis aged - high myopia; aged - PDR; aged CRVO; uveitis - high myopia; uveitis - PDR; high myopia - PDR; high myopia - BRVO; high myopia - CRVO; PDR - NPDR; PDR - CRVO.

$\mathrm{p}<0.001$; aged - BRVO.

$\mathrm{p}<0.005 ;$ aged - NPDR, aged - high myopia; aged - PDR; aged - BRVO; PDR - BRVO

$\mathrm{p}<0.01 ;$ aged - BRVO; high myopia - NPDR; BRVO - CRVO

$\mathrm{p}<0.05$; uveitis - BRVO; uveitis - NPDR.

$\mathrm{p}>0.1$; BRVO - NPDR; NPDR - CRVO.

$\mathrm{p}>0.5$; uveitis - CRVO.

diabetes glucose binds the collagen and induces collagen fibril crosslinking and aggregation with concomitant liquefaction. ${ }^{132324}$ Vitreous shrinkage frequently is observed in disease related vitreous change. Blood components and cell mediation are thought to be the primary mechanisms of vitreous shrinkage in disease related PVD. ${ }^{25-28}$ Free radical induced crosslinking of collagen fibres also is thought a cause vitreous shrinkage. ${ }^{29}{ }^{30}$ The mechanism of weakening of vitreoretinal adhesion is uncertain. Recent reports have suggested that changes in the extracellular matrix combined with the thickness of the internal limiting lamina and the hyalocytes found in the posterior vitreous cortex may be associated with changes in vitreoretinal adhesion. ${ }^{31}{ }^{32}$

Complete PVD with collapse is typical of the age related type, which is characterised by reduced vitreoretinal adhesion, vitreous gel liquefaction, and mild vitreous shrinkage. Highly myopic eyes also frequently have complete PVD with collapse. Diffuse chorioretinal atrophy in high myopia might induce vitreous liquefaction and somehow reduce vitreoretinal adhesion.

Complete PVD without collapse was most frequently associated with uveitis and central retinal vein occlusion in the present study. Increased blood components or inflammatory cells induce vitreous gel shrinkage. The vitreous gel did not exhibit as much liquefaction because these patients are younger. Vitreous shrinkage induces PVD with minimal vitreous liquefaction, resulting in complete PVD without collapse in these patients.

Partial PVD with TPVC typically was observed in association with proliferative diabetic retinopathy, in which new vessels grow out of the retina and the optic disc into the vitreous cortex. ${ }^{33}$ Strong adhesion results from cell migration and proliferation in the vitreous. Chronic leakage of the blood components induces vitreous shrinkage, especially in the posterior vitreous cortex in these cases. The concurrence of strong vitreoretinal adhesion and vitreous shrinkage produces this kind of PVD. Clinically, detecting this type of PVD is very important. In diabetic retinopathy, it is a precursor of tractional retinal detachment.
Partial PVD without TPVC was found mostly in patients without retinal disease. This may be a transitory phase of complete PVD with collapse or another result of age related vitreous change, because the difference in patient ages between complete PVD with collapse and this type was not significant. Two types of partial PVD without TPVC were found in the present study, that in which the posterior vitreous cortex is not taut (Fig 4) and in which the vitreous gel is attached to the macular area through a round defect in the posterior vitreous cortex (Fig 5). The latter type may be easily misdiagnosed as complete PVD unless the examiner carefully scans the posterior fundus. This type of PVD is more common than the former type and has to be diagnosed correctly, ${ }^{96-19}$ because vitreous attachment is an important factor affecting the prognosis of macular diseases such as macular breaks ${ }^{34-36}$ or preretinal membranes. ${ }^{18} 37$

In summary, our new clinical classification of PVD is precise and corresponds well with the presence of various types of retinal disease. This classification of PVD might be useful for evaluating vitreoretinal disorders.

1 Davis MD. Vitreous contraction in proliferative diabetic retinopathy. Arch Ophthalmol 1965;74:741-51.

2 Tolentino FI, Lee PF, Schepens CL. Biomicroscopic study of vitreous cavity in diabetic retinopathy. Arch Ophthalmol 1966;75:238-46.

3 Jalkh AE, Takahashi M, Topilow HW, Trempe CL, McMeel JW. Prognostic value of vitreous findings in diabetic retinopathy. Arch Ophthalmol 1982;100:432-4.

4 Takahashi M, Trempe CL, Maguire K, McMeel JW. Vitreoretinal relationship in diabetic retinopathy. A biomicroscopic evaluation. Arch Ophthalmol 1981;99:241-5.

5 Goldmann H. Senescenz des Glaskörpers. Ophthalmologica 1962;143:253-79.

6 Eisner G. Zur anatomie des Glaskörpers. Graefes Arch Clin Exp Ophthalmol 1975;193:33-56.

7 O'Malley C. The pattern of vitreous syneresis - a study of 800 autopsy eyes. In: Irvine AR, O'Malley C, eds. Advances in vitreous surgery. Springfield, IL: Charles C Thomas, 1976:17-33.

8 Tolentino FI, Schepens CL, Freeman HM. Vitreoretinal disorders, diagnosis and management. Philadelphia: WB Saunders, 1976:130-50.

9 Jaffe NS. Vitreous traction at the posterior pole of the fundus due to alterations in the vitreous posterior. Trans Am Acad Ophthalmol Otolaryngol 1967;71:642-52.

10 Balazs EA, Denlinger JL. Aging changes in the vitreous. In: Sekuler R, Kline D, Dismukes K, eds. Aging and human visual function. New York: Alan R Liss, 1982:45-57.

11 Foos RY, Wheeler NC. Vitreoretinal juncture, synchisis senilis and posterior vitreous detachment. Ophthalmology 1982;891502-12.

12 Larsson L, Osterlin S. Posterior vitreous detachment-a combined clinical and physiochemical study. Graefes Arch Clin Exp Ophthalmol 1985;223:92-5. 
13 Sebag J, Buckingham BB, Charles MA, Reiser K. Biochemi$\mathrm{cal}$ abnormalities in vitreous of humans with proliferative $\mathrm{cal}$ abnormalities in vitreous of humans with proliferative

14 Takahashi M, Trempe CL, Schepens CL. Biomicroscopic evaluation and photography of posterior vitreous detachevaluation and photography of posterior
ment. Arch Ophthalmol 1980;98:665-8.

15 Kakehashi A, Akiba J, Trempe CL. Vitreous photography with a +90-diopter double aspheric preset lens vs the El Bayadi-Kajiura preset lens. Arch Ophthalmol 1991;109: $962-5$.

16 Sebag J, Balazs EA. Pathogenesis of cystoid macular edema: An anatomic consideration of vitreoretinal adhesions. Surv Ophthalmol 1984;28(suppl):493-8.

17 Sebag J, Balazs EA. Human vitreous fibers and vitreoretinal disease. Trans Ophthalmol Soc UK 1985;104:123-8.

18 Kakehashi A, Trempe CL, Schepens CL. Vitreomacular pathology. I. Vitreomacular adhesions and premacular hole in hyaloid. Ophthalmology 1994;101:1515-21.

19 Kakehashi A, Ishiko S, Konno S, Kado M, Akiba J, Yoshida A. Observing the posterior vitreous by means of the A. Observing the posterior vitreous by means of the 113:558-60.

20 Ueno N, Sebag J, Hirokawa H, Chakrabarti B. Effects of visible-light irradiation of vitreous structure in the presence of a photosensitizer. Exp Eye Res 1987;44:863-70.

21 Ueno N, Chakrabarti B. Liquefaction of human vitreous by $\mathrm{N}$-formylkynurenine-type photosensitizer in model aphakic eyes: monitoring liquefaction by fluorescence. Curr Eye Res 1990;9:487-92.

22 Chattopadhyaya D, Akiba J, Ueno N, Chakrabarti B. Metal ion-catalyzed liquefaction of vitreous by ascorbic acid: role of radicals and radical ions. Ophthalmic Res 1992;24:1-7.

23 Sebag J. Abnormalities of human vitreous structure in diabetes. Graefes Arch Clin Exp Ophthalmol 1993;231:25760.

24 Sebag J, Nie S, Reiser K, Charles MA, Yu Nai-Teng. Raman spectroscopy of human vitreous in proliferative diabetic retinopathy. Invest Ophthalmol Vis Sci 1994;35:2976-80.
25 Akiba J, Ueno N, Chakrabarti B. Molecular mechanisms of posterior vitreous detachment. Graefes Arch Clin Exp Ophthalmol 1993;231:408-12.

26 Akiba J, Kakehashi A, Ueno N, Tano Y, Chakrabarti B. Serum-induced collagen gel contraction. Graefes Arch Clin Exp Ophthalmol 1995;233:430-4.

27 Akiba J, Kado M, Kakehashi A, Trempe CL. Role of the vitreous in posterior segment neovascularization in central retinal vein occlusion. Ophthalmic Surg 1991;22:498-502.

28 Glaser BM. Pathology of PVR. In: Freeman HM, Tolentino FI, eds. Proliferative vitreoretinopathy (PVR). New York: Springer-Verlag, 1988:12-21.

29 Kakehashi A, Akiba J, Ueno N, Chakrabarti B. Evidence for singlet oxygen-induced cross-links and aggregation of collagen. Biochem Biophys Res Commun 1993;196:1440-6.

30 Kakehashi A, Ueno N, Chakrabarti B. Molecular mechanism of photochemically induced posterior vitreous detachment. Ophthalmic Res 1994;26:51-9.

31 Sebag J. Age-related differences in the human vitreoretinal interface. Arch Ophthalmol 1991;109:966-71.

32 Kohno T, Sorgente N, Ishibashi T, Goodnight R, Ryan SJ Immunofluorescent studies of fibronectin and laminin in the human eye. Invest Ophthalmol Vis Sci 1987;28:506-14.

33 Faulborn J, Bowald S. Microproliferations in proliferative diabetic retinopathy and their relationship to the vitreous: corresponding light and electron microscopic studies. Graefes Arch Clin Exp Ophthalmol 1985;223:130-8.

34 Gass JDM. Idiopathic senile macular hole: its early stages and pathogenesis. Arch Ophthalmol 1988;106:629-39.

35 Akiba J, Yoshida A, Trempe CL. Risk of developing a macular hole. Arch Ophthalmol 1990;108:1088-90.

36 Akiba J, Kakehashi A, Arzabe C, Trempe CL. Prognosis of fellow eye in idiopathic macular hole cases. Ophthalmic Surg 1992;23:594-7.

37 Hirokawa H, Jalkh A, Takahashi M, Takahashi M, Trempe CL, Schepens CL. Role of vitreous in idiopathic preretinal macular fibrosis. Arch Ophthalmol 1986;101:166-9. 